CUBO A Mathematical Journal

Vol.12, $N^{\underline{Q}}$ 01, (83-93). March 2010

\title{
A Fixed Point Theorem of Reich in $G$-Metric Spaces
}

\author{
Zead Mustafa And Hamed OBiedat \\ The Hashemite University, Department of Mathematics, \\ P.O. Box 150459, Zarqa 13115, Jordan \\ emails: zmagablh@hu.edu.jo, \\ hobiedat@hu.edu.jo
}

\begin{abstract}
In this paper we prove some fixed point results for mapping satisfying sufficient contractive conditions on a complete $G$-metric space, also we showed that if the $G$-metric space $(X, G)$ is symmetric, then the existence and uniqueness of these fixed point results follows from Reich theorems in usual metric space $\left(X, d_{G}\right)$, where $\left(X, d_{G}\right)$ the metric induced by the $G$-metric space $(X, G)$.
\end{abstract}

\section{RESUMEN}

En este artículo nosotros provamos algunos resultados de punto fijo para aplicaciones satisfaciendo condiciones suficientes de contractividad sobre un espacio $G$-métrico completo, también mostramos que si el espacio $G$-métrico $(X, G)$ es simétrico, entonces la existencia y unicidad de estos resultados de punto fijo siguen de teoremas de Reich en espacios métricos usuales $\left(X, d_{G}\right)$, donde $\left(X, d_{G}\right)$ es la métrica inducida por el espacio $G$-métrico $(X, G)$.

Key words and phrases: Metric space, generalized metric space, D-metric space, 2-metric space.

Math. Subj. Class.: 47H10, 46B20. 


\section{Introduction}

The study of fixed points of a functions satisfying certain contractive conditions has been at the center of vigorous research activity, because it has a wide range of applications in different areas such as, variational, linear inequalities, optimization and parameterize estimation problems.

In 2005, Z. Mustafa and B. Sims introduced a new class of generalized metric spaces (see [2], $[3])$, which are called $G$-metric spaces as generalization of metric space $(X, d)$, to develop and to introduce a new fixed point theory for a variety of mappings in this new setting, also to extend known metric space theorems to a more general setting. The $G$-metric space is as follows.

Definition 1. Let $X$ be a nonempty set, and let $G: X \times X \times X \rightarrow \boldsymbol{R}^{+}$, be a function satisfying the following properties:

(G1) $G(x, y, z)=0$ if $x=y=z$;

(G2) $0<G(x, x, y)$; for all $x, y \in X$, with $x \neq y$;

(G3) $G(x, x, y) \leq G(x, y, z)$, for all $x, y, z \in X$, with $z \neq y$;

(G4) $G(x, y, z)=G(x, z, y)=G(y, z, x)=\ldots$, (symmetry in all three variables); and

(G5) $G(x, y, z) \leq G(x, a, a)+G(a, y, z)$, for all $x, y, z, a \in X$, (rectangle inequality).

Then the function $G$ is called a generalized metric, or, more specifically a $G$-metric on $X$, and the pair $(X, G)$ is called a $G$-metric space.

Example 1. ([2]) Let $(X, d)$ be a usual metric space, and define $G_{s}$ and $G_{m}$ on $X \times X \times X$ to $\mathbf{R}^{+}$by

$$
\begin{gathered}
G_{s}(x, y, z)=d(x, y)+d(y, z)+d(x, z), \text { and } \\
G_{m}(x, y, z)=\max \{d(x, y), d(y, z), d(x, z)\}
\end{gathered}
$$

for all $x, y, z \in X$. Then $\left(X, G_{s}\right)$ and $\left(X, G_{m}\right)$ are $G$-metric spaces.

Definition 2. ([3]) Let $(X, G)$ be a G-metric space, and let $\left(x_{n}\right)$ be a sequence of points of $X$. A point $x \in X$ is said to be the limit of the sequence $\left(x_{n}\right)$ if $\lim _{n, m \rightarrow \infty} G\left(x, x_{n}, x_{m}\right)=0$, and one say that the sequence $\left(x_{n}\right)$ is $G$-convergent to $x$.

Thus, that if $x_{n} \longrightarrow 0$ in a $G$-metric space $(X, G)$, then for any $\epsilon>0$, there exists $N \in \mathbf{N}$ such that $G\left(x, x_{n}, x_{m}\right)<\epsilon$, for all $n, m \geq N$, (we mean by $\mathbf{N}$ the Natural numbers).

Proposition 1. ([3]) Let $(X, G)$ be $G$-metric space. Then the following are equivalent.

(1) $\quad\left(x_{n}\right)$ is G-convergent to $x$. 
(3) $\quad G\left(x_{n}, x_{n}, x\right) \rightarrow 0$, as $n \rightarrow \infty$.

(4) $G\left(x_{n}, x, x\right) \rightarrow 0$, as $n \rightarrow \infty$.

(5) $\quad G\left(x_{m}, x_{n}, x\right) \rightarrow 0$, as $m, n \rightarrow \infty$.

Definition 3. ([3]) Let $(X, G)$ be a G-metric space, a sequence $\left(x_{n}\right)$ is called $G$-Cauchy if given $\epsilon>0$, there is $N \in \mathbf{N}$ such that $G\left(x_{n}, x_{m}, x_{l}\right)<\epsilon$, for all $n, m, l \geq N$. That is $G\left(x_{n}, x_{m}, x_{l}\right) \longrightarrow 0$ as $n, m, l \longrightarrow \infty$.

Proposition 2. ([3]) In a G-metric space, $(X, G)$, the following are equivalent.

1. The sequence $\left(x_{n}\right)$ is G-Cauchy.

2. For every $\epsilon>0$, there exists $N \in \mathbf{N}$ such that $G\left(x_{n}, x_{m}, x_{m}\right)<\epsilon$, for all $n, m \geq N$.

Definition 4. ([3]) Let $(X, G)$ and $\left(X^{\prime}, G^{\prime}\right)$ be two G-metric spaces, and let $f:(X, G) \rightarrow\left(X^{\prime}, G^{\prime}\right)$ be a function, then $f$ is said to be $G$-continuous at a point $a \in X$ if and only if, given $\epsilon>0$, there exists $\delta>0$ such that $x, y \in X$; and $G(a, x, y)<\delta$ implies $G^{\prime}(f(a), f(x), f(y))<\epsilon$. A function $f$ is $G$-continuous at $X$ if and only if it is $G$-continuous at all $a \in X$.

Proposition 3. ([3]) Let $(X, G)$, and $\left(X^{\prime}, G^{\prime}\right)$ be two $G$-metric spaces. Then a function $f: X \longrightarrow$ $X^{\prime}$ is $G$-continuous at a point $x \in X$ if and only if it is $G$-sequentially continuous at $x$; that is, whenever $\left(x_{n}\right)$ is $G$-convergent to $x$ we have $\left(f\left(x_{n}\right)\right)$ is $G$-convergent to $f(x)$.

Definition 5. ([3]) A G-metric space $(X, G)$ is called symmetric $G$-metric space if $G(x, y, y)=$ $G(y, x, x)$ for all $x, y \in X$.

It is clear that, any $G$-metric space where $G$ derives from an underlying metric via $\mathrm{G}_{s}$ or $\mathrm{G}_{m}$ in Example 1 is symmetric.

The following example presents the simplest instance of a nonsymmetric $G$-metric and so also one which does not arise from any metric in the above ways.

Example 2. ([3]) Let $X=\{a, b\}$, and let,

$$
G(a, a, a)=G(b, b, b)=0
$$

$G(a, a, b)=1, G(a, b, b)=2$

and extend $G$ to $X \times X \times X$ by symmetry in the variables. Then it is easily verified that $G$ is a $G$-metric, but $G(a, b, b) \neq G(a, a, b)$.

Proposition 4. ([3]) Let $(X, G)$ be a G-metric space, then the function $G(x, y, z)$ is jointly continuous in all three of its variables.

Proposition 5. ([3]) Every $G$-metric space $(X, G)$ induces a metric space $\left(X, d_{G}\right)$ defined by

$$
d_{G}(x, y)=G(x, y, y)+G(y, x, x), \forall x, y \in X .
$$


Note that if $(X, G)$ is symmetric, then

$$
d_{G}(x, y)=2 G(x, y, y), \forall x, y \in X
$$

However, if $(X, G)$ is not symmetric then it holds by the $G$-metric properties that

$$
\frac{3}{2} G(x, y, y) \leq d_{G}(x, y) \leq 3 G(x, y, y), \forall x, y \in X
$$

Definition 6. ([3]) A $G$-metric space $(X, G)$ is said to be $G$-complete ( or complete $G$-metric ) if every $G$-Cauchy sequence in $(X, G)$ is $G$-convergent in $(X, G)$.

Proposition 6. ([3])A G-metric space $(X, G)$ is $G$-complete if and only if $\left(X, d_{G}\right)$ is a complete metric space.

Theorem 1.1 (Reich,[4]). Let $(X, d)$ be a complete metric space, and $T$ be a function mapping $X$ into it self, satisfy the following condition,

$$
d(T(x), T(y)) \leq a d(x, T(x))+b d(y, T(y))+c d(x, y), \forall x, y \in X
$$

where $a, b, c$ are nonnegative numbers satisfying $a+b+c<1$.

Then, $T$ has a unique fixed point (i.e., there exists $u \in X ; T u=u$ ).

\section{Main Results}

In this section, we will present several fixed point results on a complete $G$-metric space.

Theorem 2.1. Let $(X, G)$ be a complete $G$-metric space, and let $T: X \longrightarrow X$ be a mapping satisfies the following condition

$$
G(T(x), T(y), T(z)) \leq k\{G(x, T(x), T(x))+G(y, T(y), T(y))+G(z, T(z), T(z))\}
$$

for all $x, y, z \in X$, where $k \in[0,1 / 3)$. Then $T$ has a unique fixed point (say $u$ ), and $T$ is $G$ continuous at $u$.

Proof. Suppose that $T$ satisfies condition (2.1), then for all $x, y \in X$, we have

$$
\begin{gathered}
G(T x, T y, T y) \leq k[G(x, T x, T x)+2 G(y, T y, T y)], \text { and } \\
G(T y, T x, T x) \leq k[G(y, T y, T y)+2 G(x, T x, T x)] .
\end{gathered}
$$

Suppose that $(X, G)$ is symmetric. Then from the definition of metric $\left(X, d_{G}\right)$ and $(1.1)$, we have

$$
d_{G}(T x, T y) \leq k d_{G}(x, T x)+2 k d_{G}(y, T y), \forall x, y \in X
$$


In this line, since $0<k+2 k<1$, then the metric condition (2.4) will be a special case of the Reich condition (1.3), so the existence and uniqueness of the fixed point follows from Theorem (1.1).

However, if $(X, G)$ is not symmetric then we can conclude that $d_{G}(T x, T y)=G(T x, T y, T y)+G(T y, T x, T x) \leq 3 k G(x, T x, T x)+3 k G(y, T y, T y)$, $\forall x, y \in X$.

So, by the definition of the metric $\left(X, d_{G}\right)$ and $(1.2)$, we get

$$
d_{G}(T x, T y) \leq 2 k d_{G}(x, T x)+2 k d_{G}(y, T y), \forall x, y \in X,
$$

and, the metric condition gives no information about this map since $0<2 k+2 k$ need not be less than 1. But the existence of a fixed point can be proved using properties of a $G$-metric.

Let $x_{0} \in X$, be an arbitrary point, and define the sequence $\left(x_{n}\right)$ by $x_{n}=T^{n}\left(x_{0}\right)$, then the condition (2.1) implies that

$G\left(x_{n}, x_{n+1}, x_{n+1}\right) \leq k G\left(x_{n-1}, x_{n}, x_{n}\right)+2 k G\left(x_{n}, x_{n+1}, x_{n+1}\right)$, hence

$$
G\left(x_{n}, x_{n+1}, x_{n+1}\right) \leq \frac{k}{1-2 k} G\left(x_{n-1}, x_{n}, x_{n}\right) .
$$

Let $q=\frac{k}{1-2 k}$, then $0<q<1$ since $0 \leq k<1 / 3$.

So,

$$
G\left(x_{n}, x_{n+1}, x_{n+1}\right) \leq q G\left(x_{n-1}, x_{n}, x_{n}\right) .
$$

Continuing in the same argument, we will find

$$
G\left(x_{n}, x_{n+1}, x_{n+1}\right) \leq q^{n} G\left(x_{0}, x_{1}, x_{1}\right) .
$$

Moreover, for all $n, m \in \mathbf{N} ; n<m$ we have by repeated use the rectangle inequality and using equation (2.5) that

$G\left(x_{n}, x_{m}, x_{m}\right) \leq G\left(x_{n}, x_{n+1}, x_{n+1}\right)+G\left(x_{n+1}, x_{n+2}, x_{n+2}\right)$

$+G\left(x_{n+2}, x_{n+3}, x_{n+3}\right)+\ldots+G\left(x_{m-1}, x_{m}, x_{m}\right)$

$\leq\left(q^{n}+q^{n+1}+\ldots+q^{m-1}\right) G\left(x_{0}, x_{1}, x_{1}\right) \leq \frac{q^{n}}{1-q} G\left(x_{0}, x_{1}, x_{1}\right)$,

and so, $\lim G\left(x_{n}, x_{m}, x_{m}\right)=0$, as $n, m \longrightarrow \infty$. Thus $\left(x_{n}\right)$ is $G$-Cauchy sequence, then by completeness of $(X, G)$, there exists $u \in X$ such that $\left(x_{n}\right)$ is $G$-convergent to $u$.

Assume on the contrary that $T(u) \neq u$. Then

$G\left(x_{n+1}, T(u), T(u)\right) \leq k\left\{G\left(x_{n}, x_{n+1}, x_{n+1}\right)+2 G(u, T(u), T(u))\right\}$.

Taking the limit as $n \longrightarrow \infty$, and using the fact that the function $G$ is continuous on its variable, this leads to $G(u, T(u), T(u)) \leq 2 k G(u, T(u), T(u))$. This contradiction implies that $u=T(u)$.

To prove uniqueness, suppose that $u$ and $v$ are two fixed points for $T$, then $G(u, v, v) \leq k G(u, T(u), T(u))+2 k G(v, T(v), T(v))=0$, which implies that $u=v$. 
To show that $T$ is $G$-continuous at $u$, let $\left(y_{n}\right) \subseteq X$ be a sequence converges to $u$ in $(X, G)$, then we can deduce that

$$
G\left(u, T\left(y_{n}\right), T\left(y_{n}\right)\right) \leq k\left\{G(u, T(u), T(u))+2 G\left(y_{n}, T\left(y_{n}\right), T\left(y_{n}\right)\right)\right\} .
$$

Moreover, from $G$-metric axioms we have,

$$
G\left(y_{n}, T\left(y_{n}\right), T\left(y_{n}\right)\right) \leq G\left(y_{n}, u, u\right)+G\left(u, T\left(y_{n}\right), T\left(y_{n}\right)\right),
$$

so, equation (2.6) implies that $G\left(u, T\left(y_{n}\right), T\left(y_{n}\right)\right) \leq \frac{2 k}{1-2 k} G\left(y_{n}, u, u\right)$. Taking the limit as $n \rightarrow \infty$, from which we see that $G\left(y_{n}, T\left(y_{n}\right), T\left(y_{n}\right)\right) \rightarrow 0$ and so, by Proposition 3, $T\left(y_{n}\right) \rightarrow u=T u$, therefor $T$ is $G$-continuous at $u$. This completes the prove of Theorem (2.1).

Corollary 1. Let $(X, G)$ be a complete $G$-metric spaces, and let $T: X \longrightarrow X$ be a mapping satisfying the following condition for some $m \in \mathbf{N}$

$$
G\left(T^{m}(x), T^{m}(y), T^{m}(z)\right) \leq k\left\{\begin{array}{l}
G\left(x, T^{m}(x), T^{m}(x)\right)+G\left(y, T^{m}(y), T^{m}(y)\right)+ \\
G\left(z, T^{m}(z), T^{m}(z)\right)
\end{array}\right\}
$$

for all $x, y, z \in X$, where $k \in[0,1 / 3)$. Then $T$ has unique fixed point (say $u$ ), and $T^{m}$ is $G$ continuous at $u$.

Proof. From previous theorem we see that $T^{m}$ has a unique fixed point (say u), that is, $T^{m}(u)=u$, and $T^{m}(u)$ is $G$-continuous at $u$. But, $T(u)=T\left(T^{m}(u)\right)=T^{m+1}(u)=T^{m}(T(u))$, so $T(u)$ is another fixed point for $T^{m}$ and by uniqueness $T u=u$.

Theorem 2.2. Let $(X, G)$ be a complete $G$-metric space, and let $T: X \longrightarrow X$, be a mapping satisfying the following condition

$$
G(T(x), T(y), T(z)) \leq \alpha G(x, y, z)+\beta\left\{\begin{array}{l}
G(y, T(y), T(y))+G(z, T(z), T(z))+ \\
G(x, T(x), T(x))
\end{array}\right\}
$$

for all $x, y, z \in X$, where $0 \leq \alpha+3 \beta<1$. Then $T$ has unique fixed point (say u), and $T$ is $G$-continuous at $u$.

Proof. Suppose that $T$ satisfies condition (2.8). Then for all $x, y \in X$

$$
\begin{gathered}
G(T x, T y, T y) \leq \alpha G(x, y, y)+\beta[G(x, T x, T x)+2 G(y, T y, T y)], \text { and } \\
G(T y, T x, T x) \leq \alpha G(y, x, x)+\beta[G(y, T y, T y)+2 G(x, T x, T x)] .
\end{gathered}
$$

Suppose that $(X, G)$ is symmetric. Then from the definition of metric $\left(X, d_{G}\right)$ and (1.1) we get

$$
d_{G}(T x, T y) \leq \alpha d_{G}(x, y)+\beta d_{G}(x, T x)+2 \beta d_{G}(y, T y), \forall x, y \in X .
$$


Since $0<\alpha+3 \beta<1$, then the metric condition (2.11) becomes the same as Reich condition (1.3), so the existence and uniqueness of the fixed point follows from Theorem (1.1).

However, if $(X, G)$ is not symmetric, then we conclude that $d_{G}(T x, T y)=G(T x, T y, T y)+G(T y, T x, T x) \leq$ $\alpha[G(x, y, y)+G(y, x, x)]+3 \beta G(x, T x, T x)+3 \beta G(y, T y, T y), \forall x, y \in X$.

So, by the definition of the metric $\left(X, d_{G}\right)$ and $(1.2)$ we get

$$
d_{G}(T x, T y) \leq \alpha d_{G}(x, y)+2 \beta d_{G}(x, T x)+2 \beta d_{G}(y, T y), \forall x, y \in X
$$

The metric condition gives no information about this map since $0<\alpha+2 \beta+2 \beta$ need not be less 1 , but this can be proved by $G$-metric.

Let $x_{0} \in X$, be an arbitrary point, and define the sequence $\left(x_{n}\right)$ by $x_{n}=T^{n}\left(x_{0}\right)$, then by (2.8) we can verify that

$G\left(x_{n}, x_{n+1}, x_{n+1}\right) \leq \alpha G\left(x_{n-1}, x_{n}, x_{n}\right)+\beta\left\{G\left(x_{n-1}, x_{n}, x_{n}\right)+2 G\left(x_{n}, x_{n+1}, x_{n+1}\right)\right\}$ then

$$
(1-2 \beta) G\left(x_{n}, x_{n+1}, x_{n+1}\right) \leq(\alpha+\beta) G\left(x_{n-1}, x_{n}, x_{n}\right)
$$

therefore,

$$
G\left(x_{n}, x_{n+1}, x_{n+1}\right) \leq \frac{\alpha+\beta}{1-2 \beta} G\left(x_{n-1}, x_{n}, x_{n}\right) .
$$

Let $q=\frac{\alpha+\beta}{1-2 \beta}$, then $0 \leq q<1$ since $0 \leq \alpha+3 \beta<1$.

So,

$$
G\left(x_{n}, x_{n+1}, x_{n+1}\right) \leq q G\left(x_{n-1}, x_{n}, x_{n}\right) .
$$

Continuing in the same argument, we will find

$$
G\left(x_{n}, x_{n+1}, x_{n+1}\right) \leq q^{n} G\left(x_{0}, x_{1}, x_{1}\right) .
$$

For all $n, m \in \mathbf{N} ; n<m$, we have by repeated use the rectangle inequality that

$G\left(x_{n}, x_{m}, x_{m}\right) \leq G\left(x_{n}, x_{n+1}, x_{n+1}\right)+G\left(x_{n+1}, x_{n+2}, x_{n+2}\right)$

$+G\left(x_{n+2}, x_{n+3}, x_{n+3}\right)+\ldots+G\left(x_{m-1}, x_{m}, x_{m}\right)$

$\leq\left(q^{n}+q^{n+1}+\ldots+q^{m-1}\right) G\left(x_{0}, x_{1}, x_{1}\right) \leq \frac{q^{n}}{1-q} G\left(x_{0}, x_{1}, x_{1}\right)$.

Then, $\lim G\left(x_{n}, x_{m}, x_{m}\right)=0$, as $n, m \longrightarrow \infty$, thus $\left(x_{n}\right)$ is $G$-Cauchy sequence. Due to the completeness of $(X, G)$, there exists $u \in X$ such that $\left(x_{n}\right)$ is $G$-convergent to $u$ in $(X, G)$.

Suppose that $T(u) \neq u$. Then

$G\left(x_{n}, T(u), T(u)\right) \leq \alpha G\left(x_{n-1}, u, u\right)+\beta\left\{G\left(x_{n-1}, x_{n}, x_{n}\right)+2 G(u, T(u), T(u))\right\}$,

and by taking the limit as $n \longrightarrow \infty$, and using the fact that the function $G$ is continuous, we get that $G(u, T(u), T(u)) \leq 2 \beta G(u, T(u), T(u))$. This contradiction implies that $u=T(u)$.

To prove uniqueness, suppose that $u$ and $v$ are two fixed points for $T$. Then $G(u, v, v) \leq \alpha G(u, v, v)+\beta\{G(u, T(u), T(u))+2 \beta G(v, T(v), T(v))\}=0+\alpha G(u, v, v)$, which is implies that $u=v$, since $0<\alpha<1$. 
To show that $T$ is $G$-continuous at $u$, let $\left(y_{n}\right) \subseteq X$ be a sequence converges to $u$ in $(X, G)$, then we deduce that

$$
\begin{aligned}
G\left(u, T\left(y_{n}\right), T\left(y_{n}\right)\right) & \leq \alpha G\left(u, y_{n}, y_{n}\right)+\beta\left\{G(u, T(u), T(u))+2 G\left(y_{n}, T\left(y_{n}\right), T\left(y_{n}\right)\right)\right\} \\
& =\alpha G\left(u, y_{n}, y_{n}\right)+2 \beta G\left(y_{n}, T\left(y_{n}\right), T\left(y_{n}\right)\right) .
\end{aligned}
$$

But, by $G$-metric axioms we have

$G\left(y_{n}, T\left(y_{n}\right), T\left(y_{n}\right)\right) \leq G\left(y_{n}, u, u\right)+G\left(u, T\left(y_{n}\right), T\left(y_{n}\right)\right)$,

so equation (2.12) implies that

$G\left(u, T\left(y_{n}\right), T\left(y_{n}\right)\right) \leq \frac{\alpha}{1-2 \beta} G\left(u, y_{n}, y_{n}\right)+\frac{2 \beta}{1-2 \beta} G\left(y_{n}, u, u\right)$. Taking the limit as $n \rightarrow \infty$, from which we see that $G\left(y_{n}, T\left(y_{n}\right), T\left(y_{n}\right)\right) \rightarrow 0$, and so by Proposition $3, T\left(y_{n}\right) \rightarrow u=T u$. So, $T$ is $G$-continuous at $u$. This completes the proof of Theorem (2.2).

Corollary 2. Let $(X, G)$ be a complete $G$-metric spaces, and let $T: X \longrightarrow X$ be a mapping satisfying, the following condition for some $m \in \mathbf{N}$

$$
G\left(T^{m}(x), T^{m}(y), T^{m}(z)\right) \leq \alpha G(x, y, z)+\beta\left\{\begin{array}{c}
G\left(x, T^{m}(x), T^{m}(x)\right)+ \\
G\left(y, T^{m}(y), T^{m}(y)\right)+ \\
G\left(z, T^{m}(z), T^{m}(z)\right)
\end{array}\right\}
$$

for all $x, y, z \in X$, where $0 \leq \alpha+3 \beta<1$. Then $T$ has unique fixed point (say $u$ ), and $T^{m}$ is $G$-continuous at $u$.

Proof. We use the same argument in Corollary 1.

Theorem 2.3. Let $(X, G)$ be complete $G$-metric space, and let $T: X \longrightarrow X$ be a mapping satisfying the condition

$$
G(T(x), T(y), T(z)) \leq \alpha G(x, y, z)+\beta \max \left\{\begin{array}{l}
G(x, T(x), T(x)), G(y, T(y), T(y)), \\
G(z, T(z), T(z))
\end{array}\right\}
$$

for all $x, y, z \in X$, where $0 \leq \alpha+\beta<1$. Then $T$ has unique fixed point (say $u$ ), and $T$ is $G$-continuous at $u$.

Proof. Suppose that $T$ satisfies condition (2.14). Then for all $x, y \in X$

$$
\begin{gathered}
G(T x, T y, T y) \leq \alpha G(x, y, y)+\beta \max \{G(x, T x, T x), G(y, T y, T y)\}, \text { and } \\
G(T y, T x, T x) \leq \alpha G(y, x, x)+\beta \max \{G(y, T y, T y),(x, T x, T x)\} .
\end{gathered}
$$

Suppose that $(X, G)$ is symmetric. Then from the definition of metric $\left(X, d_{G}\right)$ and (1.1) we get.

$$
d_{G}(T x, T y) \leq \alpha d_{G}(x, y)+\beta \max \left\{d_{G}(x, T x), d_{G}(y, T y)\right\}, \forall x, y \in X .
$$


In this line since $0 \leq \alpha+\beta<1$, then the metric condition (2.17) will be a special case of the Reich condition (1.3). Therefor the existence and uniqueness of the fixed point follows from Theorem (1.1).

However, if $(X, G)$ is not symmetric then

$$
d_{G}(x, y)=G(T x, T y, T y)+G(T y, T x, T x) \leq \alpha[G(x, y, y)+G(y, x, x)]+
$$
$2 \beta \max \{G(x, T x, T x), G(y, T y, T y)\}$.

So, by definition of the metric $\left(X, d_{G}\right)$ and $(1.2)$, we will have

$$
d_{G}(T x, T y) \leq \alpha d_{G}(x, y)+2 \beta \max \left\{\frac{2}{3} d_{G}(x, T x), \frac{2}{3} d_{G}(y, T y)\right\}, \forall x, y \in X .
$$

The metric condition gives no information about this map since $\alpha+\frac{4 \beta}{3}$ need not be less than 1 . But the existence of a fixed point can be proved using properties of a $G$-metric.

Let $x_{0} \in X$, be arbitrary point, and define the sequence $\left(x_{n}\right)$ by $x_{n}=T^{n}\left(x_{0}\right)$, then by $(2.14)$ we get.

$$
G\left(x_{n}, x_{n+1}, x_{n+1}\right) \leq \alpha G\left(x_{n-1}, x_{n}, x_{n}\right)+\beta \max \left\{G\left(x_{n-1}, x_{n}, x_{n}\right), G\left(x_{n}, x_{n+1}, x_{n+1}\right)\right\} .
$$

We see that there are two cases :

(1) Suppose $\max \left\{G\left(x_{n-1}, x_{n}, x_{n}\right), G\left(x_{n}, x_{n+1}, x_{n+1}\right)\right\}=G\left(x_{n-1}, x_{n}, x_{n}\right)$. Then in this case, equation (2.18) implies that

$$
G\left(x_{n}, x_{n+1}, x_{n+1}\right) \leq(\alpha+\beta) G\left(x_{n-1}, x_{n}, x_{n}\right) .
$$

(2) Suppose $\max \left\{G\left(x_{n-1}, x_{n}, x_{n}\right), G\left(x_{n}, x_{n+1}, x_{n+1}\right)\right\}=G\left(x_{n}, x_{n+1}, x_{n+1}\right)$. Then in this case, equation (2.18) implies that

$$
\begin{gathered}
G\left(x_{n}, x_{n+1}, x_{n+1}\right) \leq \alpha G\left(x_{n-1}, x_{n}, x_{n}\right)+\beta G\left(x_{n}, x_{n+1}, x_{n+1}\right), \text { therefore } \\
\\
G\left(x_{n}, x_{n+1}, x_{n+1}\right) \leq \frac{\alpha}{1-\beta} G\left(x_{n-1}, x_{n}, x_{n}\right),
\end{gathered}
$$

but in this case we have $G\left(x_{n-1}, x_{n}, x_{n}\right) \leq G\left(x_{n}, x_{n+1}, x_{n+1}\right)$, hence

$$
G\left(x_{n-1}, x_{n}, x_{n}\right) \leq G\left(x_{n}, x_{n+1}, x_{n+1}\right) \leq \frac{\alpha}{1-\beta} G\left(x_{n-1}, x_{n}, x_{n}\right)
$$

which is a contradiction since $\frac{\alpha}{1-\beta}<1$.

Then, it must be the case (1) is true, which says that

$$
G\left(x_{n}, x_{n+1}, x_{n+1}\right) \leq(\alpha+\beta) G\left(x_{n-1}, x_{n}, x_{n}\right) .
$$

Let $q=\alpha+\beta$, then $0 \leq q<1$ since $0 \leq \alpha+\beta<1$, therefor

$$
G\left(x_{n}, x_{n+1}, x_{n+1}\right) \leq q G\left(x_{n-1}, x_{n}, x_{n}\right) .
$$


Continuing in the same argument, we will find

$$
G\left(x_{n}, x_{n+1}, x_{n+1}\right) \leq q^{n} G\left(x_{0}, x_{1}, x_{1}\right) .
$$

For all $n, m \in \mathbf{N} ; n<m$ we have by repeated use the rectangle inequality that $G\left(x_{n}, x_{m}, x_{m}\right) \leq$ $G\left(x_{n}, x_{n+1}, x_{n+1}\right)+G\left(x_{n+1}, x_{n+2}, x_{n+2}\right)$

$+G\left(x_{n+2}, x_{n+3}, x_{n+3}\right)+\ldots+G\left(x_{m-1}, x_{m}, x_{m}\right)$

$\leq\left(q^{n}+q^{n+1}+\ldots+q^{m-1}\right) G\left(x_{0}, x_{1}, x_{1}\right) \leq \frac{q^{n}}{1-q} G\left(x_{0}, x_{1}, x_{1}\right)$.

This proved that, $\lim G\left(x_{n}, x_{m}, x_{m}\right)=0$, as $n, m \longrightarrow \infty$, thus $\left(x_{n}\right)$ is $G$-Cauchy sequence. Due to the completeness of $(X, G)$, there exists $u \in X$ such that $\left(x_{n}\right)$ is $G$-convergent to $u$ in $(x, G)$.

Suppose that $T(u) \neq u$. Then by condition (2.14), we have

$$
G\left(x_{n}, T(u), T(u)\right) \leq \alpha G\left(x_{n-1}, u, u\right)+\beta \max \left\{G\left(x_{n-1}, x_{n}, x_{n}\right), G(u, T(u), T(u))\right\} .
$$

Taking the limit as $n \longrightarrow \infty$, and using the fact that the function $G$ is continuous, we get $G(u, T(u), T(u)) \leq \beta G(u, T(u), T(u))$, this contradiction implies that $u=T(u)$.

To prove uniqueness, suppose that $u$ and $v$ are two fixed points for $T$. Then by (2.14) we have

$G(u, v, v) \leq \alpha G(u, v, v)+\beta \max \{G(u, T(u), T(u)), G(v, T(v), T(v))\}=\alpha G(u, v, v)$, since $\alpha<1$ this implies that $u=v$.

To show that $T$ is $G$-continuous at $u$, let $\left(y_{n}\right) \subseteq X$ be a sequence converging to $u$ in $(X, G)$, then

$$
\begin{gathered}
G\left(u, T\left(y_{n}\right), T\left(y_{n}\right)\right) \leq \alpha G\left(u, y_{n}, y_{n}\right)+\beta \max \left\{G(u, T(u), T(u)), G\left(y_{n}, T\left(y_{n}\right), T\left(y_{n}\right)\right)\right\}, \text { hence } \\
G\left(u, T\left(y_{n}\right), T\left(y_{n}\right)\right) \leq \alpha G\left(u, y_{n}, y_{n}\right)+\beta G\left(y_{n}, T\left(y_{n}\right), T\left(y_{n}\right)\right)
\end{gathered}
$$

But, by $G$-metric axioms we have

$G\left(y_{n}, T\left(y_{n}\right), T\left(y_{n}\right)\right) \leq G\left(y_{n}, u, u\right)+G\left(u, T\left(y_{n}\right), T\left(y_{n}\right)\right)$.

Thus equation (2.19) implies that,

$G\left(u, T\left(y_{n}\right), T\left(y_{n}\right)\right) \leq \frac{\alpha}{1-\beta} G\left(u, y_{n}, y_{n}\right)+\frac{\beta}{1-\beta} G\left(y_{n}, u, u\right)$.

Taking the limit as $n \rightarrow \infty$, from which we see that

$G\left(u, T\left(y_{n}\right), T\left(y_{n}\right)\right) \rightarrow 0$ and so, by Proposition (3), we have $T\left(y_{n}\right) \rightarrow u=T u$ which implies that $T$ is $G$-continuous at $u$. This completes the proof of Theorem (2.3).

Corollary 3. Let $(X, G)$ be a complete $G$-metric spaces, and let $T: X \longrightarrow X$ be a mapping satisfying the following condition for some $m \in \mathbf{N}$

$$
G\left(T^{m}(x), T^{m}(y), T^{m}(z)\right) \leq \alpha G(x, y, z)+\beta \max \left\{\begin{array}{c}
G\left(x, T^{m}(x), T^{m}(x)\right), \\
G\left(y, T^{m}(y), T^{m}(y)\right), \\
G\left(z, T^{m}(z), T^{m}(z)\right)
\end{array}\right\}
$$

for all $x, y, z \in X$, where $0 \leq \alpha+\beta<1$. Then $T$ has unique fixed point (say u), and $T^{m}$ is $G$-continuous at $u$. 
Proof. We use the same argument in Corollary 1.

$$
\text { Received: September, 2008. Revised: October, } 2009 .
$$

\section{References}

[1] Mustafa, Z. and Sims, B., Some Remarks Concerninig D-Metric Spaces, Proceedings of the Internatinal Conferences on Fixed Point Theorey and Applications, Valencia (Spain), July (2003). 189-198.

[2] Mustafa, Z., A New Structure For Generalized Metric Spaces - With Applications To Fixed Point Theory, PhD Thesis, the University of Newcastle, Australia, 2005.

[3] Mustafa, Z. and Sims, B., A New Approach to Generalized Metric Spaces, Journal of Nonlinear and Convex Analysis, Volume 7, No. 2 (2006). 289-297.

[4] Reich, S., Some Remarks concerning contraction mappings, Canad. Math. Bull. 14, (1971), $121-124$. MR $49 \sharp 1501$. 